\title{
Efficient Calculation of the Green's Functions for Multilayered Shielded Cavities with Right Isosceles-Triangular Cross-Section
}

\author{
J.S. Gomez-Diaz, M. Martinez-Mendoza, F.D. Quesada-Pereira, \\ and A. Alvarez-Melcon *
}

October 14, 2008

\begin{abstract}
In this paper, an efficient calculation of the Green's functions inside multilayered shielded cavities with right isosceles-triangular cross-section is presented. The method is entirely developed in the spatial domain, and it is based on image theory. The idea is to use the spatial domain Green's functions inside a multilayered shielded square box, in order to accurately obtain the Green's functions for the right isosceles triangular cavity. Image theory is then used to enforce the boundary conditions along the non-equal side of the triangle. It is shown that the new algorithm is very robust, with limited computational effort. Resonance frequencies and potential patterns of a triangular cavity have been calculated and compared to those obtain by other techniques, showing very good agreement. Finally, a transversal filter inside a multilayered triangular shaped cavity is designed, manufactured and tested using the developed technique.
\end{abstract}

\section{Introduction}

The analysis of shielded microwave circuits is an interesting subject that has always attracted attention. This is because they find huge applications in many modern telecommunication systems. In order to achieve high accuracy, full-wave analysis must be performed to analyze microwave devices. This is the case for example of modern monolithic microwave integrated circuits (MMIC). The effects of the shielding enclosure can not be neglected, due to their influence in the electrical behavior of the whole structure [1].

For the analysis of these devices, finite-elements methods can be employed (such as [2] or [3]). However, these family of methods are computationally very heavy and time-consuming. Other possi-

\footnotetext{
${ }^{*}$ Technical University of Cartagena. Campus Muralla del Mar, s/n, E-30202 Cartagena, Spain.
} 
bility is to employ the integral equation technique (IE) combined with the Method of Moments (MoM) [4].

In the latter case, the main problem is to compute the Green's functions associated with the multilayered shielded medium. This calculation can be performed in the spectral or in the spatial domains. The spectral domain is usually very efficient, although it has some convergence problems when the shielding box has large dimensions as compared to the size of the printed circuit. Rectangular [5] and circular [6] multilayered enclosures have efficiently been treated in the spectral domain. On the other hand, the spatial domain is able to solve some of the problems encountered in the spectral domain, but still expresses the Green's functions in terms of slow convergent series of spatial images. Rectangular enclosures have extensively been analyzed in this domain (see [7] or [8]).

Recently, a new technique based on equivalent point sources has been developed to numerically compute the Green's functions in circular cavities [9] and in arbitrarily-shaped cavities defined by linear segments [10], using an spatial formulation. These previous approaches were useful approximations to the calculation of the exact Green's functions for these kind of cavities. In the process, the weights of the fictitious sources are numerically computed to impose the boundary conditions at discrete points on the cavity wall. This imposition of the boundary conditions needs also to be done along the longitudinal direction, for electrically long cavities.

In this paper, we present a new rigorous approach for the Green's functions calculation inside a multilayered shielded cavity with right isosceles triangular cross-section. The method is entirely developed in the spatial domain and it is based on image theory. Unlike the previous techniques ([9],[10]), the method computes the Green's functions rigorously in the whole cavity, without introducing equivalent sources. The key idea is to split a multilayered square shaped box in two right isosceles triangular cavities (namely A and B as shown in Fig. 1). We then use the spatial domain Green's functions associated to the square cavity, to recover the Green's functions related to one of the triangular structures. Note that the Green's functions of square cavities can be efficiently computed by using series acceleration techniques [11]. Once the Green's functions of the square cavity are computed, simple image theory is used to enforce the boundary conditions along the non-equal side of the triangle. In this way, for each point source inside (A), a spatial image will be placed in the other triangular cavity, (B). This image is able to exactly satisfy the boundary condition along the hypotenuse side of the triangular cavity, even along the longitudinal direction (height of the cavity). Finally, the Green's functions inside the triangle (A) are recovered by taking into account the presence of the original and of the image sources.

As in the works presented in [9] and [10], this paper uses the concept of spatial images related to the electric scalar and magnetic vector potentials. However, it is important to remark that the present 
approach exactly satisfies the boundary conditions along the whole cavity wall (even along the height of the enclosure), and therefore it is not an approximation to the modeling of the enclosure. Furthermore, the images are computed using the Green's functions associated to a multilayered shielded square cavity, instead of the usual multilayered infinite medium employed in previous works. On the other hand, the use of this rigorous approach is restricted to the case of multilayered enclosures with triangular right-isosceles cross section.

The usefulness of the technique is demonstrated by obtaining resonance frequencies and potentials patterns of a multilayered cavity with right isosceles triangular cross-section. The results are compared with those obtained by a finite-elements method (HFSS ${ }^{\circledR}$ ), showing good agreement. In order to show the practical value of the method, a hybrid waveguide-microstrip filter [12], inside a triangular cavity, is designed using the developed technique. Since this filter combines one of the cavity resonances with a resonance of a printed microstrip line, the correct model of the triangular-shaped multilayered cavity is essential. Measured data is used to validate the filter results.

\section{Theoretical Outline}

The geometry for the calculation of the mixed-potential Green's function is presented in Fig. 1. As it can be seen in the figure, an electric unitary dipole is placed inside a right-isosceles triangular metallic cavity (A). The final goal is to compute the Green's functions associated to this triangular cavity (A). To do that, a square cavity, which is composed of the original triangle (A) and of an auxiliary triangle (B) is considered. The Green's functions associated to a multilayered shielded square enclosure, as presented in Fig. 1, can efficiently be obtained in the spatial domain using, for instance, the procedure described in [13] or in [11]. These square cavity Green's functions are then used to recover the Green's functions associated to the multilayered triangular cavity (A). To do that, an electric point source $\left(q_{e}\right)$ or an electric unitary dipole $(I)$ is placed inside this cavity (A) (see Fig. 2 and Fig. 3) Then, the triangle (B) is used to place an exact image of the original source $\left(q_{e}^{\prime}\right.$ or $\left.I^{\prime}\right)$. Both original and image sources are computed using the square cavity Green's functions, and therefore, the boundary conditions at the external square walls are automatically satisfied. Finally, the original and image sources are combined to compute the Green's function associated to the triangular cavity (A). Due to the combination of the original source with its image, the field will also satisfy the boundary conditions at the hypotenuse of the triangular cavity. Since the original and image sources are computed inside a multilayered structure, the final computed Green's functions will also take into account for the substrate layers.

The physical boundary condition to be imposed at the metallic cavity walls is the zero tangent electric field. This boundary condition can also be translated to the potentials. First, the electric 
scalar potential must vanish along the cavity walls. If a single electric point charge is placed inside the triangle A, the boundary condition will be automatically satisfied along the two external equal sides of the triangular cavity. This is because these two sides are common to the square cavity used to calculate the basic Green's functions. In order to impose the boundary conditions along the hypotenuse of the triangle, a spatial image of the original source is placed inside triangle B (see Fig. 2). From basic image theory [14], the value of this new image is the same as the original, but with opposite sign. The combination of both sources will make the electric scalar potential to be zero along the hypotenuse of the triangle, even in the $z$-direction. Since the image also shares the square cavity sides, the boundary conditions are also satisfied in the other walls. Finally, the electric scalar potential inside the triangular cavity is recovered using a combination of the two sources:

$$
G_{V_{T}}\left(\vec{r}, \vec{r}^{\prime}\right)=G_{V_{q}}\left(\vec{r}, \vec{r}^{\prime}\right)-G_{V_{q}^{\prime}}\left(\vec{r}, \vec{r}_{i m}^{\prime}\right)
$$

where $\vec{r}$ is an arbitrary observation point $(P), \vec{r}^{\prime}$ is the source position (both inside triangle A), and $\vec{r}_{i m}^{\prime}$ is the source image position (placed in triangle B). It is important to remark that $G_{V_{q}}$ and $G_{V_{q}^{\prime}}$ are the electric scalar potential inside a square multilayered shielded cavity containing the triangular cavity.

For the evaluation of the magnetic vector potential dyadic Green's function, a similar procedure is followed, but taking into account the vector nature of this potential. Considering two unit dipoles inside triangle $\mathrm{A}$ ( $I_{x}$ and $I_{y}$, oriented along the $x$ and $y$ axis), two images dipoles can be placed inside triangle $\mathrm{B}\left(I_{x}^{\prime}\right.$ and $I_{y}^{\prime}$, oriented along the $y$ and $x$-axis respectively). These images are used to enforce the boundary conditions along the triangle hypotenuse (see Fig. 3). Again, since all dipoles are placed inside the associated square cavity, the boundary conditions are also imposed along the external walls. By simple image theory, the values of the image dipoles are the same as the original, but their orientations have been rotated by 90 degrees [14]. The final magnetic vector potential is recovered using the superposition of all dipoles:

$$
\begin{aligned}
& G_{A_{T}}^{x x}\left(\vec{r}, \vec{r}^{\prime}\right)=G_{A_{I_{x}}}^{x x}\left(\vec{r}, \vec{r}^{\prime}\right) \\
& G_{A_{T}}^{y x}\left(\vec{r}, \vec{r}^{\prime}\right)=G_{A_{I_{x}^{\prime}}}^{y y}\left(\vec{r}, \vec{r}^{\prime}{ }_{i m}\right) \\
& G_{A_{T}}^{x y}\left(\vec{r}, \vec{r}^{\prime}\right)=-G_{A_{I_{y}^{\prime}}}^{x x}\left(\vec{r}, \vec{r}^{\prime}{ }_{i m}\right) \\
& G_{A_{T}}^{y y}\left(\vec{r}, \vec{r}^{\prime}\right)=G_{A_{I_{y}}}^{y y}\left(\vec{r}, \vec{r}^{\prime}\right)
\end{aligned}
$$

where $\vec{r}$ is the position of an arbitrary observation point $(P), \vec{r}^{\prime}$ is the source position (both inside triangle A), and $\vec{r}_{i m}^{\prime}$ is the source images position (placed in triangle B). It is worth mentioning that, according to these expressions, an $x$-directed dipole will produce a $y$-component of the magnetic vector potential (and viceversa). This cross component is given by the image dipole $I_{x}^{\prime}$, which is oriented along the $y$-axis. Physically, this cross component is caused by the hypotenuse wall of the triangular 
cavity.

The computational effort required to evaluate the proposed Green's functions for multilayered triangular cavities represents twice the effort required for the computation of the Green's functions associated to multilayered square cavities, which is the key of the proposed formulation. Note that for the computation of the latter, different techniques for series acceleration, such as those proposed in [13],[11], may be applied.

\section{Results}

In order to demonstrate the usefulness of the proposed technique, the resonant frequencies and potential patterns of a multilayered triangular cavity are obtained. The results are validated by a finite elements method (Ansoft HFSS $\left.{ }^{\circledR}\right)$. Finally, the analysis and design of a transversal microstrip filter inside a triangular cavity is presented, using measured data as validation.

In Fig. 4 a multilayered shielded triangular cavity with right isosceles cross-section is shown. To obtain the resonances of the cavity, the potentials are represented as a function of frequency for a fixed position of source and observation points. In Fig. 5 sharp peaks in the potential response can be observed. These peaks are closely related to the resonant frequencies of the cavity. It is important to remark that at exactly the natural frequencies of the cavity, the value of the potentials will tend to infinity. Consequently, to find the resonant frequencies of the cavity we detect the maximum value of the potentials given by the selected frequency step during the frequency sweep. To study the accuracy of the results, the same study has been performed by a finite-elements method (HFSS $\left.{ }^{\circledR}\right)$. As it can be seen in Table 1, high accuracy has been achieved, maintaining in all cases a relative error below $0.08 \%$, when the frequency step is $0.005 \lambda$. It is important to note that the accuracy obtained directly depends on the step length taken by the frequency sweep. When this step becomes smaller, the error decreases. For example, when the frequency step is reduced to $0.0025 \lambda$, the maximum relative error is always below $0.04 \%$.

The method presented in this paper can compute the potentials (even at the cavity resonances) without any convergence problem. To show that this is indeed the case, the electric scalar potential is shown in Fig. 6 at the normalized frequency of $\frac{L^{\prime}}{\lambda}=0.2851$, where $L^{\prime}$ denotes the physical length of the two equal sides of the triangle (see Fig. 1). Furthermore, the magnetic vector potential $G_{A}^{y y}$ is depicted in Fig. 7 at the normalized frequency of $\frac{L^{\prime}}{\lambda}=0.2991$. In both cases, a noise-free potential pattern can be observed. Note that the potential values have been normalized, and the representation avoids the singular behavior found at exactly the source position. The same study has been performed using the commercial software HFSS ${ }^{\circledR}$, obtaining similar results for the $z$-component and for the $y$-component 
of the Electric Field, respectively.

Finally, in order to verify the boundary conditions for the computed Green's functions, we include in Fig. 8 a plot of the magnetic vector potential produced by a $y$-directed unitary dipole, in the same conditions as in Fig. 7. We can observe in the figure that the magnetic vector potential remains perpendicular to the three walls of the cavity, therefore fulfilling with accuracy the proper boundary conditions.

The proposed technique can be useful to analyze microwave filters inside multilayered triangular shaped cavities, as shown in Fig. 9. This filter is a hybrid waveguide-microstrip filter [12], which combines one of the cavity resonances with a printed line microstrip resonance in order to obtain a second order filter response. The $L^{\prime}$ dimension of the cavity (related to the physical length of the two equal sides of the triangle, see Fig. 1) is adjusted in order to tune the cavity resonance at the frequency of $4.47 \mathrm{GHz}$. The resulting filter follows the topology known as Modified Doublet [15], and it can be expressed in term of the following coupling matrix $(M)[12,16]$

$$
M=\left(\begin{array}{rrrr}
0 & -0.5249 & -0.6487 & 0.0179 \\
-0.5249 & 1.0447 & 0 & 0.5249 \\
0.6487 & 0 & -0.9477 & 0.6487 \\
0.0179 & 0.5249 & 0.6487 & 0
\end{array}\right) \text {. }
$$

After the design process, a prototype, shown in Fig. 10, has been manufactured and tested. It is important to remark that the adequate modeling of the triangular multilayered box is essential for this type of filter. This is because one of the resonances of the filter is provided by the partially filled cavity.

It is important to point out that a finite elements technique has difficulties in analyzing this filter. This is because of the tight couplings existing between the input and output ports and the printed line resonator, which is difficult to model by a volume mesh. On the contrary, with an integral equation technique these couplings can be modeled more accurately. This is shown in Fig. 11, where we present the response obtained with the new method. Measured results are also shown for validations. Good agreement between both results can be observed.

\section{Conclusions}

In this paper, a novel technique for the evaluation of the Green's function inside triangular multilayered cavities with right-isosceles cross section has been presented. The spatial domain Green's functions for a square multilayered box are used to accurately obtain the Green's function for the triangular cavity. Image theory is used to exactly enforce the boundary conditions for the potentials 
along the non-equal side of the triangular cavity. Resonance frequencies and potential patterns inside a multilayered triangular cavity are obtained and validated with a finite elements technique. In order to show the practical value of the proposed method, a novel waveguide-microstrip transversal filter has been designed, analyzed and tested. Very good agreement between the simulated and measured data has been found.

\section{References}

[1] L. P. Dunleavy and P. B. Katehi, "Shielding effects in microstrip discontinuities," IEEE Transactions on Microwave Theory and Techniques, vol. 36, no. 12, pp. 1767-1774, December 1988.

[2] J.-M. Jin and J. L. Volakis, "A finite element-boundary integral formulation for scattering by three-dimensional cavity-backed apertures," IEEE Transactions on Antennas and Propagation, vol. 39, no. 1, pp. 97-104, January 1991.

[3] M. Omiya, T. Hikage, N. Ohno, K. Horiguchi, and K. Itoh, "Design of cavity-backed slot antennas using the finite-difference time-domain technique," IEEE Transactions on Antennas and

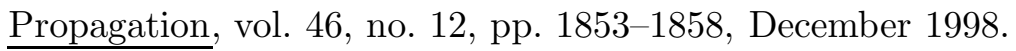

[4] R. F. Harrington, Field Computation by Moment Methods. Wiley-IEEE Press, 1968.

[5] C. J. Railton and S. A. Meade, "Fast rigorous analysis of shielded planar filters," IEEE Transactions on Microwave Theory and Techniques, vol. 40, no. 5, pp. 978-985, May 1992.

[6] F. Zavosh and J. T. Aberle, "Single and stacked circular microstrip patch antennas backed by a circular cavity," IEEE Transactions on Antennas and Propagation, vol. 43, no. 7, pp. 746-750, July 1995.

[7] L. P. Dunleavy and P. B. Katehi, "A generalized method for analyzing shielded thin microstrip discontinuities," IEEE Transactions on Microwave Theory and Techniques, vol. 36, no. 12, pp. 1758-1766, December 1988.

[8] A. A. Melcon and J. R. Mosig, "A novel spatial images technique for the analysis of cavity backed antennas," ACES, Applied Computational Electromagnetics Society, vol. 14, no. 3, March 1999.

[9] F. Q. Pereira, P. V. Castejon, D. C. Rebenaque, J. P. Garcia, and A. A. Melcon, "Numerical evaluation of the Green's functions for cylindrical enclosures," IEEE Transactions on Microwave Theory and Techniques, vol. 53, no. 1, pp. 94-105, January 2005.

[10] J. G. Diaz, M. M. Mendoza, F. P. Soler, F. Q. Pereira, and A. A. Melcon, "Practical implementation of the spatial images technique for the analysis of shielded multilayered printed circuits," 


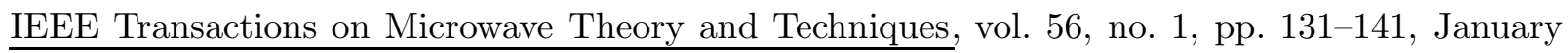
2008.

[11] M.-J. Park and S. Nam, "Efficient calculation of the green's functions for multilayered planar periodic structures," IEEE Transactions on Antennas and Propagation, vol. 46, no. 10, pp. 15821583, October 1998.

[12] M. Martínez-Mendoza, J. S. Gómez-Díaz, D. C. Rebenaque, J. L. G. Tornero, and A. AlvarezMelcon, "Design of bandpass transversal filters employing a novel hybrid structure," IEEE Transactions on Microwave Theory and Techniques, vol. 55, no. 12, pp. 2670-2678, December 2007.

[13] A. A. Melcon and J. R. Mosig, "Two techniques for the efficient numerical calculation of the Green's functions for planar shielded circuits and antennas," IEEE Transactions on Microwave Theory and Techniques, vol. 48, no. 9, pp. 1492-1504, September 2000.

[14] C. A. Balanis, Advanced Engineering Electromagnetics. John Wiley and Sons, 1989.

[15] S. Amari and U. Rosenberg, "A universal building block for advanced modular design of microwave filters," IEEE Microwave and Wireless Components Letters, vol. 13, no. 12, pp. 541-543, December 2003.

[16] R. J. Cameron, "Advanced coupling matrix synthesis techniques for microwave filters," $\underline{\text { EEE }}$ Transactions on Microwave Theory and Techniques, vol. 51, no. 1, pp. 1-10, January 2003. 
Table 1: Resonance frequencies for the triangular cavity shown in Fig. 4.

\begin{tabular}{|c|c|c|}
\hline $\begin{array}{c}\text { Resonance frequencies, } \\
\text { New Method [Ghz] }\end{array}$ & $\begin{array}{c}\text { Resonance frequencies, } \\
\text { HFSS }^{\complement}[\mathrm{Ghz}]\end{array}$ & Relative \\
\hline \hline 0.2035 & 0.2035 & 0.0000 \\
\hline 0.2210 & 0.2209 & 0.0453 \\
\hline 0.2342 & 0.2342 & 0.0000 \\
\hline 0.2585 & 0.2583 & 0.0774 \\
\hline 0.2588 & 0.2587 & 0.0387 \\
\hline 0.2695 & 0.2693 & 0.0743 \\
\hline 0.2851 & 0.2849 & 0.0702 \\
\hline 0.2991 & 0.2990 & 0.0334 \\
\hline
\end{tabular}

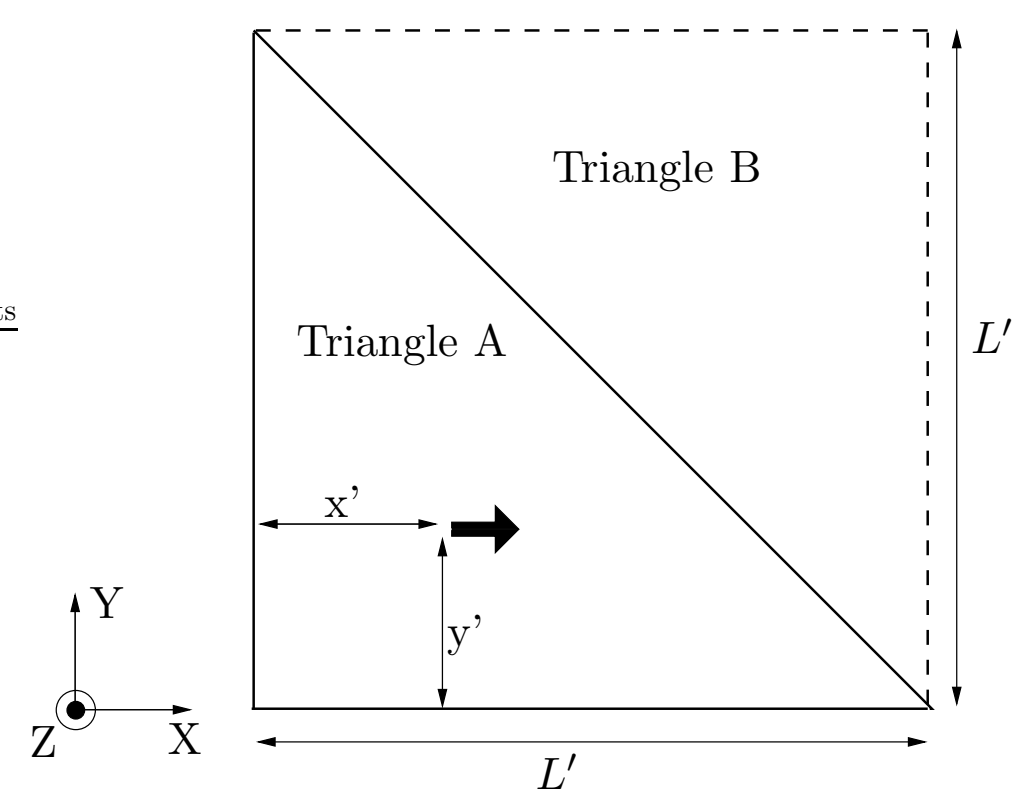

Figure 1: A square box is split in two right-isosceles triangular cavities ('A' and 'B'). A unitary electric dipole is placed inside triangle A. $L^{\prime}=\lambda, x^{\prime}=y^{\prime}=0.25 \lambda$. 


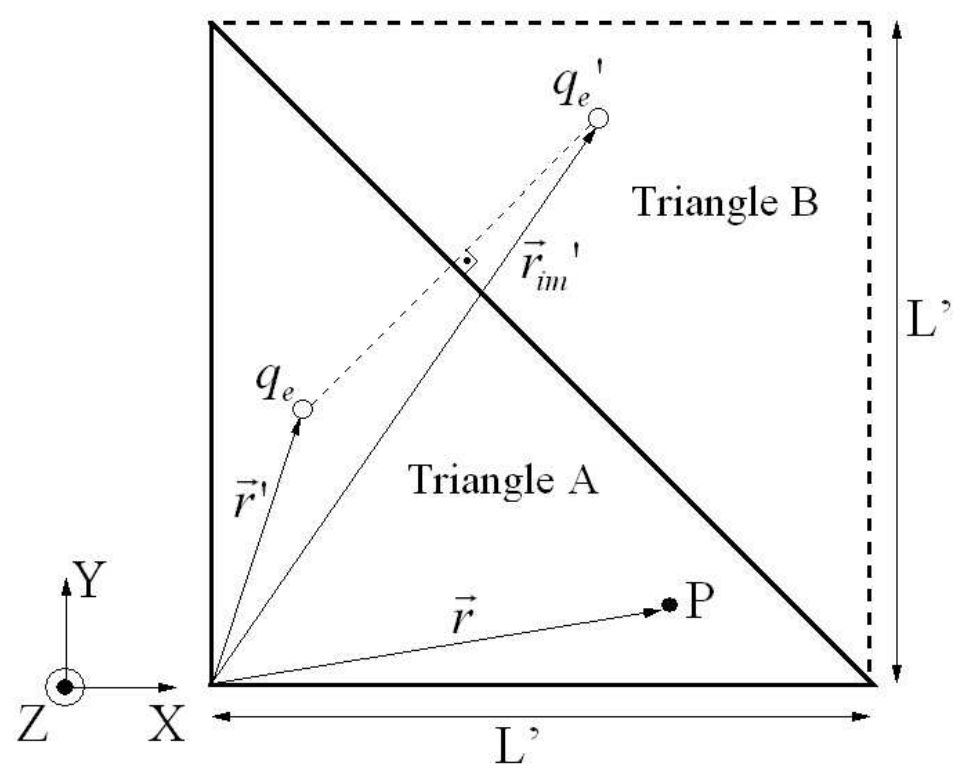

Figure 2: Original and image charge sources used to enforce the boundary condition for the electric scalar potential along the non-equal side of the triangular cavity. Point $P$ is a generic observation point. $L^{\prime}=\lambda, x^{\prime}=y^{\prime}=0.25 \lambda$.

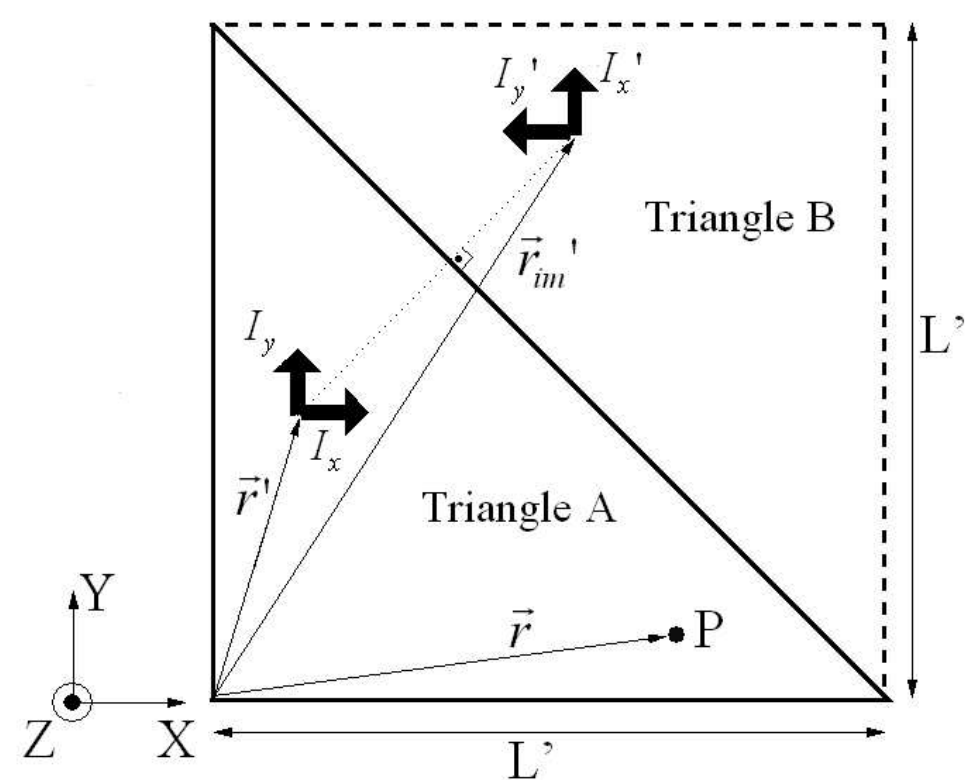

Figure 3: Original and image electric dipole sources used to enforce the boundary condition for the magnetic vector potential, along the non-equal side of the triangular cavity. Point $P$ is a generic observation point. $L^{\prime}=\lambda, x^{\prime}=y^{\prime}=0.25 \lambda$. 


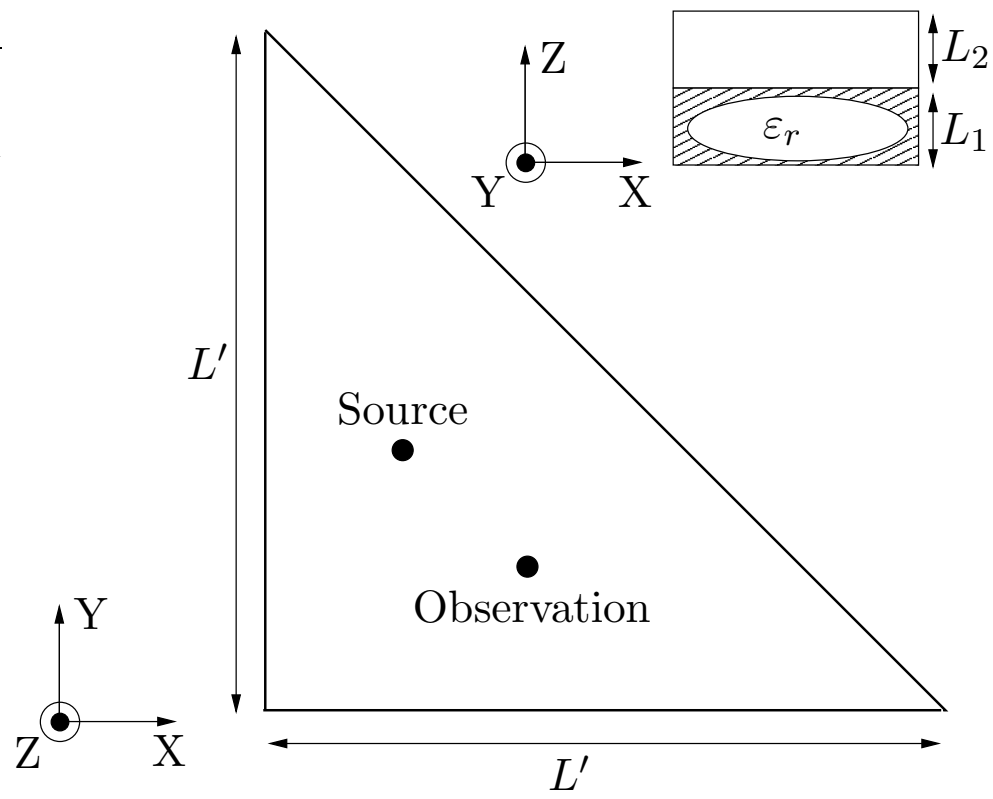

Figure 4: Multilayered shielded triangular cavity with right isosceles cross-section. $L^{\prime}=\lambda$, $L_{1}=0.2 \lambda, L_{2}=0.2 \lambda, \varepsilon_{r}=5.0$. The point source is placed at the position $(0.25 \lambda, 0.35 \lambda, 0.2 \lambda)$, and the observation point is placed at $(0.35 \lambda, 0.25 \lambda, 0.2 \lambda)$.

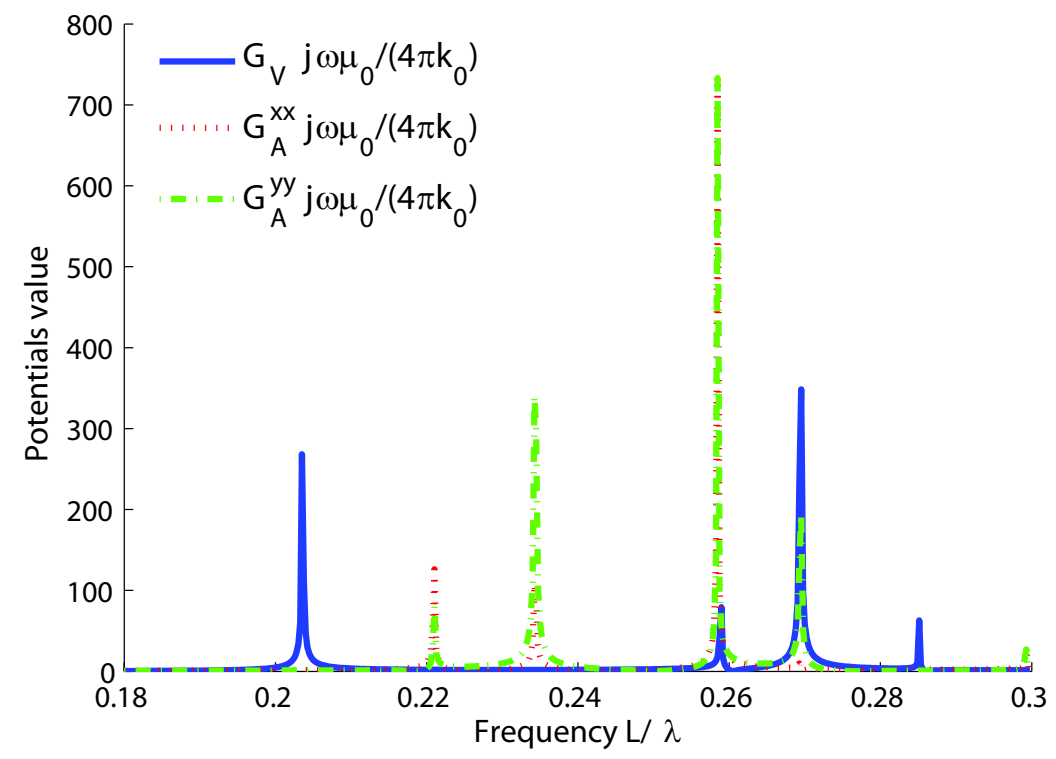

Figure 5: Mixed potentials as a function of frequency in the cavity shown in Fig. 4. 


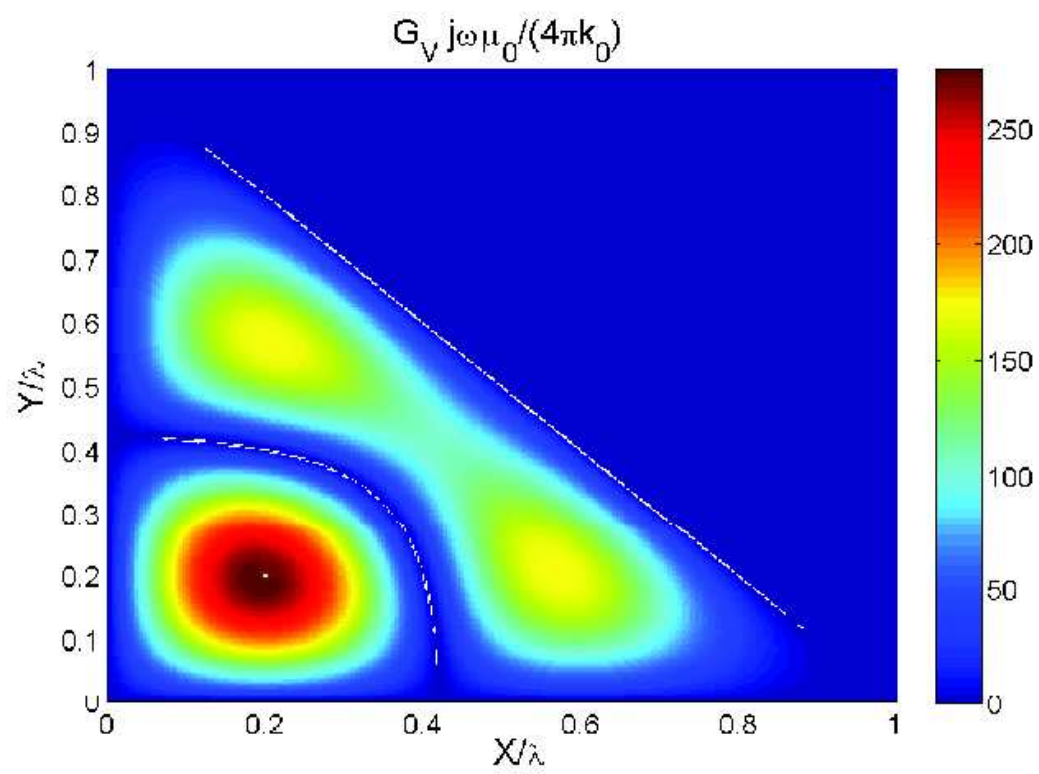

Figure 6: Electric scalar potential obtained with the new method at the normalized resonant frequency $\frac{L^{\prime}}{\lambda}=0.2851$. The source is placed at the position $(0.2 \lambda, 0.2 \lambda, 0.2 \lambda)$.

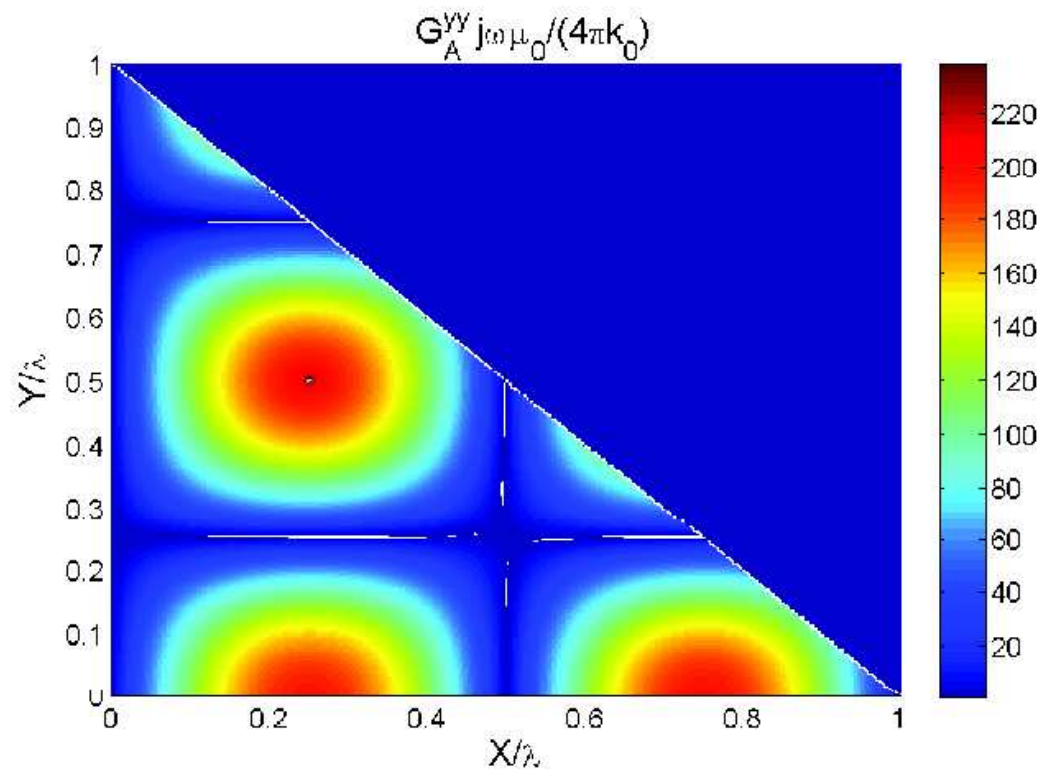

Figure 7: Magnetic vector potential dyadic component $G_{A}^{y y}$ obtained with the new method at the resonant frequency $\frac{L^{\prime}}{\lambda}=0.2991$. The source is placed at the position $(0.22 \lambda, 0.5 \lambda, 0.2 \lambda)$. 


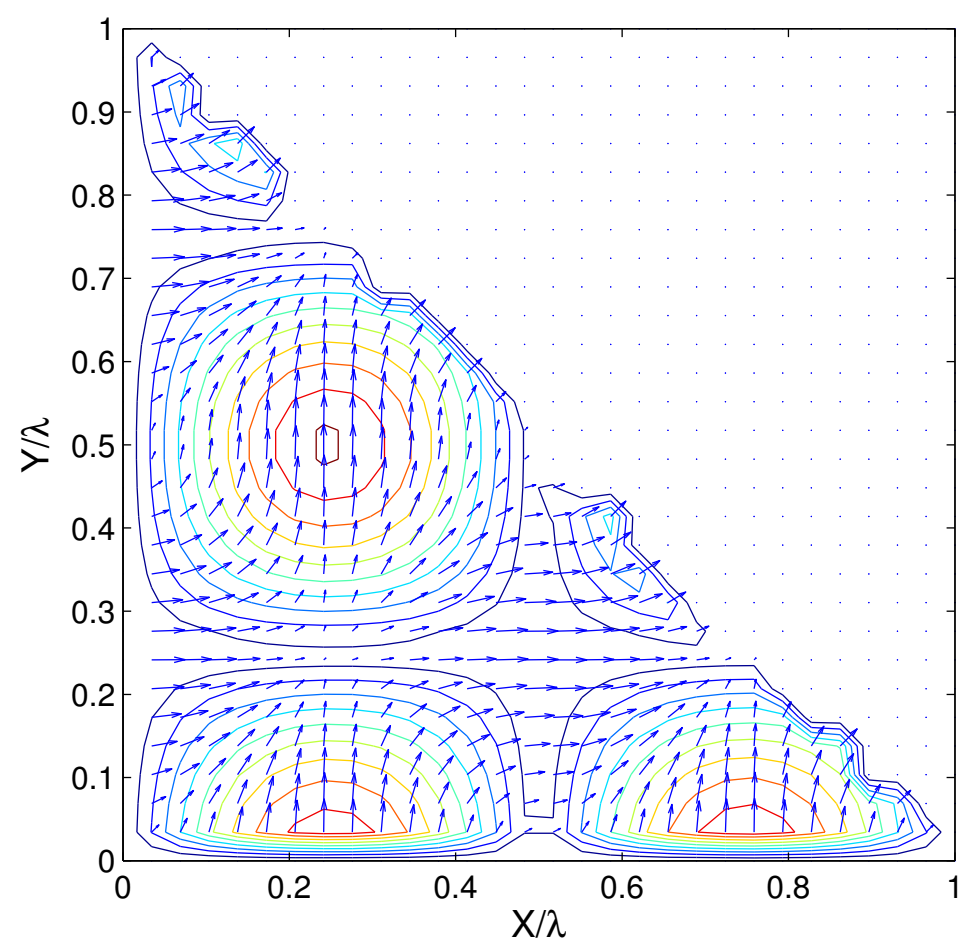

Figure 8: Vector plot of the magnetic vector potential produced by a $y$-directed unitary dipole in the same conditions as in Fig. 7.
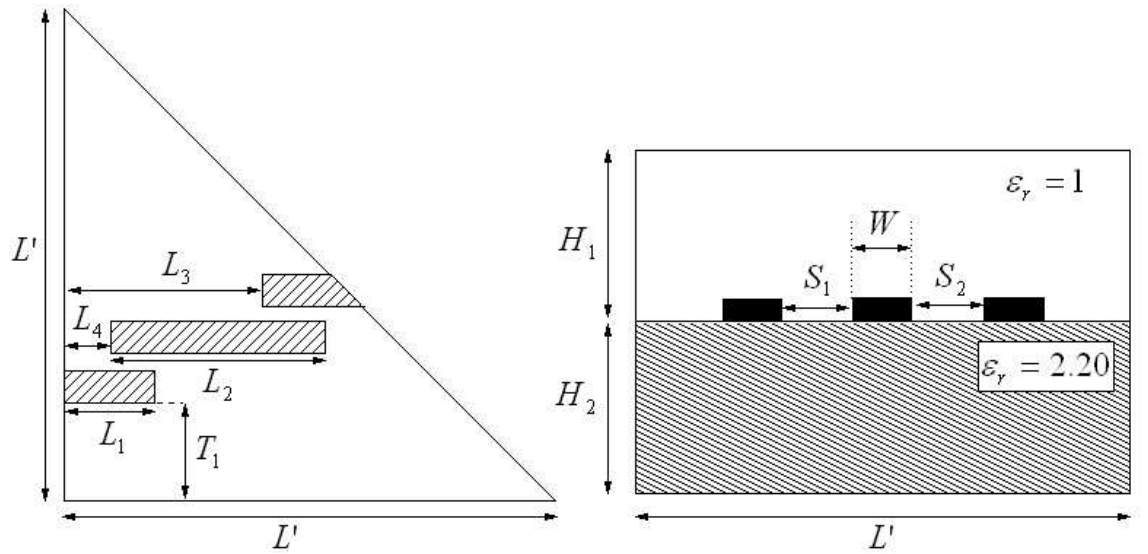

$$
\begin{array}{lllll}
L^{\prime}=63 \mathrm{~mm} & L_{1}=12.15 \mathrm{~mm} & L_{2}=23.82 \mathrm{~mm} & L_{3}=24.25 \mathrm{~mm} & L_{4}=6.39 \mathrm{~mm} \\
H_{1}=3.17 \mathrm{~mm} \quad H_{2}=2.83 \mathrm{~mm} & W=2 \mathrm{~mm} & S_{1}=S_{2}=1.5 \mathrm{~mm} & T_{1}=16.5 \mathrm{~mm}
\end{array}
$$

Figure 9: Novel triangular-shaped second order transversal filter. 


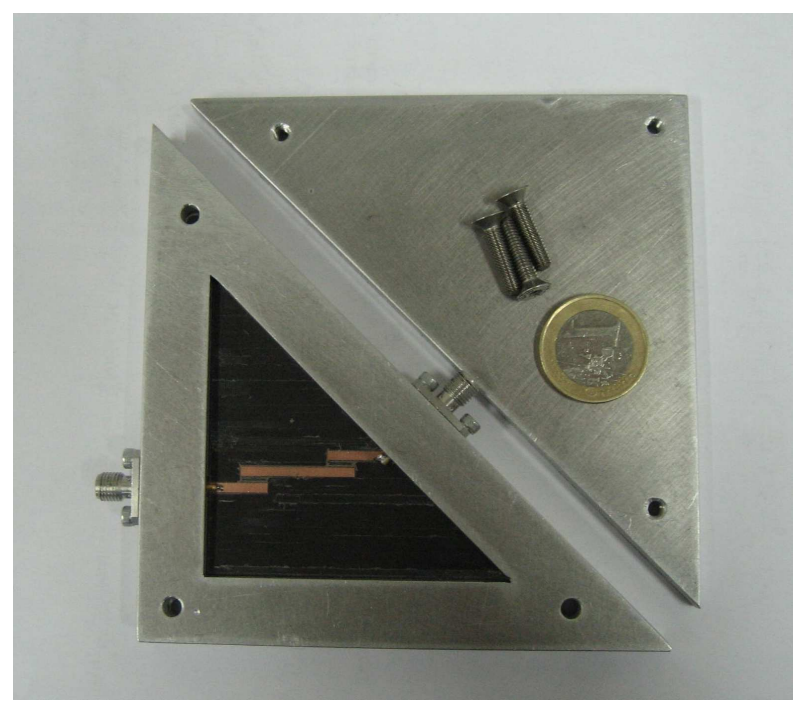

Figure 10: Aspect of the manufactured breadboard, showing all pieces of the filter.

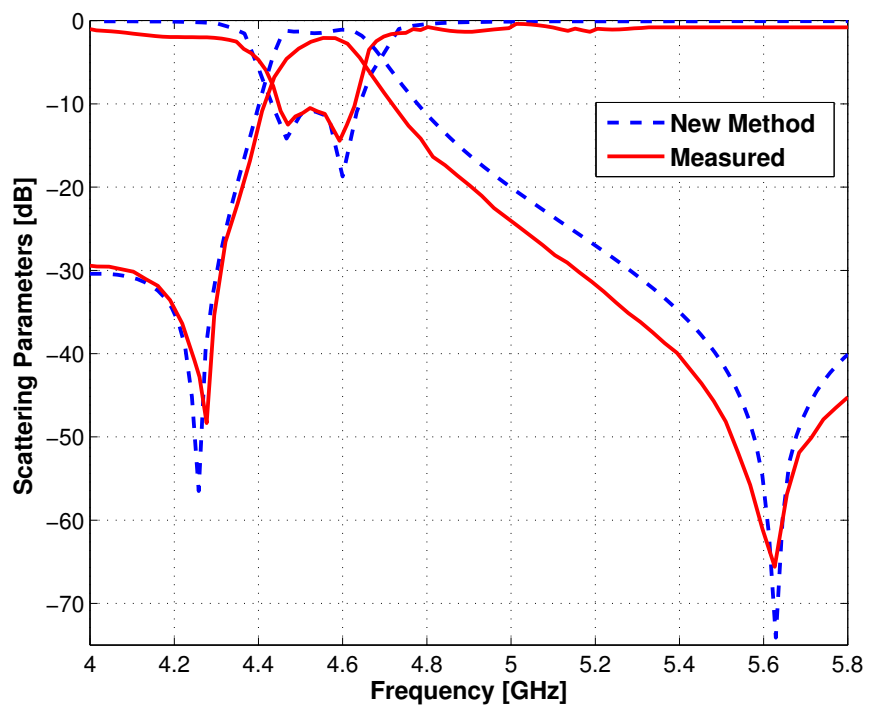

Figure 11: Results for the band-pass filter shown in Fig. 9, obtained with the proposed technique. Losses are included in the dielectric substrate $(\tan \delta=0.004)$ and in the printed metalizations $\left(\sigma=1 \cdot 10^{7} \Omega^{-1} / m\right)$. Measured data are presented as validation. 